\title{
Clinical Study of Rituximab Combined With Chemotherapy in the Treatment of Lymphoma with Hypergammaglobulinemia
}

\author{
XIAO LIN WU, GUI MIN ZHAO, CHENG HUANG, LI HONG LIU,YU HUAN GAO, HAI SHENG LIU AND RUI XIA LIU* \\ Department of Hematology, Fourth Hospital of Hebei Medical University, No.169 Tianshan Street, Shijiazhuang, Hebei \\ 050035, China
}

Wu et al.: Clinical Efficacy of Rituximab Combined with Chemotherapy in Hypergammaglobulinemia Patients

To further evaluate the clinical influencing factors of rituximab in the treatment of diffuse large $B$ cell lymphoma patients with hypergammaglobulinemia by studying the clinical efficacy of rituximab combined with chemotherapy in patients with hypergammaglobulinemia. 89 patients with diffuse large B cell lymphoma treated in our hospital from January 2011 to December 2020 were selected as subjects. After informed consent, the patients in the control group were divided into two groups: the control group was treated with conventional chemotherapy and the treatment group was treated with rituximab on the basis of conventional chemotherapy. Basic information such as age, sex, past medical history, clinical tumor stage and so on was collected. The venous blood was collected before and after treatment and the levels of hemoglobin, erythrocyte sedimentation rate, albumin, globulin, Lactate dehydrogenase and beta 2-microglobulin were measured by automatic biochemical analyzer and the levels of Immunoglobulin G, Immunoglobulin A and Immunoglobulin $M$ were measured by immunoturbidimetry. Complete remission, partial response, stable disease and disease progression, were recorded and the total effective rate was calculated. There were 27 patients in the control group (14 males and 13 females, 9 patients with hyperimmunoglobulinemia), according to Ann Arbor staging, there were 1 case in stage I, 10 cases in stage II, 7 cases in stage III and 9 cases in stage IV. There were 62 patients in the treatment group, aged $56.3 \pm 14.2 \mathrm{y}$, including 25 males and 37 females, 18 patients with hyperimmunoglobulinemia and according to Ann Arbor staging, there were 7 patients in stage I, 16 patients in stage II, 10 patients in stage III and 29 patients in stage IV. There was no significant difference in age, sex, incidence of hyperimmunoglobulinemia, International prognostic index score, tumor stage and previous history between the two groups. There was no significant difference in the levels of hemoglobin, erythrocyte sedimentation rate, albumin, lactate dehydrogenase and beta 2 -microglobulin between the control group and the treatment group before and after treatment. The erythrocyte sedimentation rate after treatment in the treatment group and the control group was lower than that before treatment and the difference was statistically significant $(p<0.05)$. There was no significant difference in the levels of Immunoglobulin G, Immunoglobulin A, ImmunoglobulinM and globulin between the control group and the treatment group before treatment, but after treatment, the level of globulin in the treatment group was significantly lower than that in the control group $(p<0.05)$. After treatment with rituximab combined with chemotherapy, the levels of ImmunoglobulinG, ImmunoglobulinA, ImmunoglobulinM and globulin in the treatment group were significantly lower than those before treatment, while the levels of ImmunoglobulinG, Immunoglobulin A and globulin in the control group were significantly lower than those before treatment, but there was no significant difference in the level of Immunoglobulin $M$ before and after treatment. After rituximab combined with chemotherapy, the total effective rate of the treatment group was $82.3 \%$, which was much higher than that of the control group $(55.6 \%)$ and the difference was statistically significant $(\mathbf{p}<0.05)$. In the treatment group, the total effective rate of patients with hypergammaglobulinemia after rituximab combined chemotherapy was $85.4 \%$, which was much higher than that in the normal group $(26.8 \%)$ and the difference was statistically significant $(\mathbf{p}<\mathbf{0 . 0 5})$. Rituximab combined with chemotherapy is effective in the treatment of diffuse large B-cell lymphoma with hypergammaglobulinemia. It can effectively reduce the level of immunoglobulin in vivo and provide reference for clinical treatment.

Key words: Rituximab, hypergammaglobulinemia, lymphoma

*Address for correspondence

E-mail: li1998abc@163.com 
There are two types of hyperimmune globulinemia (HGG), namely Polyclonal hypergammaglobulinemia (PHG) and monoclonal. The increase in monoclonal antibodies is usually due to tumor transformation of a single clone of plasma cells. During infection and inflammation, elevated serum immunoglobulin levels and obvious antibody responses can be seen. It is generally believed that this is the effect of producing antibodies from active $\mathrm{B}$ cells, which have nothing to do with accompanying infections or diseases. Infections commonly associated with hyperimmunoglobulinemia include acquired immunodeficiency syndrome, endocarditis, syphilis, and parasitic infections. Chronic diseases associated with hyperimmunoglobulinemia include systemic lupus erythematosus, cystic fibrosis and rheumatoid arthritis. Infection related polyclonal HGG is usually not involved in the disease process itself, nor is it pathological. Polyclonal HGG is most commonly associated with liver disease, malignancy, autoimmune disease or infection ${ }^{[1]}$. There are only a few studies on hypoimmunoglobulinemia in lymphoproliferative diseases. In the study of lymphoproliferative diseases with hypergammaglobulinemia, most of them are reported as individual case $\mathrm{e}^{[2-4]}$ and the number of cases is limited, mainly including hemangioimmunoblastic lymphoma and chronic lymphocytic leukemia ${ }^{[5]}$. There is no related study on lymphoma complicated with hypergamma globulinemia. Diffuse large B cell lymphoma (DLBCL) is the most common subtype of lymphoma in adults worldwide, accounting for about $1 / 3^{\text {rd }}$ of the nonHodgkin lymphomas (NHL) diagnosed each year. In clinical practice, ABVD regimen (doxorubicin+bleomycin+vincristine + dacarbazine), CHOP regimen (cyclophosphamide + doxorubicin+vincristine+prednisone) and CVP regimen (cyclophosphamide+vincristine + prednisone) were used as the traditional first-line chemotherapy regimen $^{[6]}$. In 2006, the US Food and Drug Administration (FDA) approved rituximab (Rituxmiab) in combination with conventional chemotherapy regimens for first-line treatment in patients with DLBCL, such as R-CHOP (Rituximab, Cyclophosphamide, doxorubicin, Vincristine, and Prednisone) regimens. Since then, R-CHOP has been included in the standard treatment regimen of DLBCL patients by The National Comprehensive Cancer Network $^{\circledR}\left(\mathrm{NCCN}^{\circledR}\right)$. However, it was clinically found that the efficacy of rituximab combined with traditional chemotherapy in DLBCL patients varied greatly, with the overall effective rate ranging from $72.0 \%$ to
$90.38 \%\left[0^{[7,8]}\right.$. In this paper, the clinical efficacy of rituximab combined with chemotherapy on DLBCL patients with hypergamma globulinemia will be studied to further evaluate the clinical influencing factors of rituximab in the treatment of DLBCL patients. A total of 89 patients with DLBCL admitted to our hospital from January 2011 to December 2020, with an age of $57.0 \pm 13.6 \mathrm{y}$, were selected as the research subjects, including 39 males and 50 females. Retrospective collection of patient information was made after approval by the medical ethics committee. All enrolled patients were required to sign informed consent before receiving treatment. Inclusion criteria-It met the diagnostic criteria of the Chinese Guidelines for Diagnosis and Treatment of DLBCL (2013) ${ }^{[9]}$ and was diagnosed as DLBCL by immunohistochemistry and pathological tissue diagnosis. In clinical treatment, anthracycline chemotherapy drugs were used as the basis for treatment, without the use of unknown active ingredients such as traditional Chinese medicine or Chinese patent medicine. The normal values of Immunoglobulin $\mathrm{G}$ ( $\mathrm{IgG}$ ) in vivo ranged from 7.51 to $15.6 \mathrm{~g} / \mathrm{L} . \mathrm{IgG}<7.51 \mathrm{~g} / \mathrm{L}$ was considered as hypogamma globulinemia and $\operatorname{IgG}>15.6 \mathrm{~g} / \mathrm{L}$ was considered as hypergamma globulinemia. The clinical data are complete, with complete information such as medication, diagnosis, incidence and test results during treatment. Exclusion criteria-Have an acquired immune system disease or inherited immune deficiency disease, such as lupus erythematosus, Acquired immune deficiency syndrome (AIDS) or rheumatoid arthritis. Complicated with severe organ function injury, disorder or organic lesions. Patients with mental diseases and unable to cooperate with clinical treatment. Control group-Conventional chemotherapy regimen was used and anthracycline chemotherapy drugs were used. Adjust medication appropriately according to the actual situation of patients. Treatment group-Rituximab (Shanghai Roche Pharmaceuticals Co., Ltd., National Drug Approval J20170034, $100 \mathrm{mg} / 10 \mathrm{~mL}$ ) was added on the basis of conventional chemotherapy regimen, and medication was appropriately adjusted according to the actual situation of patients. General information collection-Basic information such as age, gender, past medical history and clinical tumor stage were collected; Biochemical indicators-Venous blood was collected before and after treatment, and the levels of hemoglobin, erythrocyte sedimentation rate, albumin, globulin, LDH and $\beta 2$ microglobulin were determined by automatic biochemical analyzer; Determination of immunoglobulin levels-Venous blood was collected 
before and after treatment and IgG, IgA and IgM levels were measured by immunoturbidimetry; Clinical efficacy indicators-The criteria for judging clinical efficacy are based on the 2007 NHL International Working Group standard ${ }^{[10]}$. Complete remission (CR)clinical symptoms were eliminated or basically eliminated, abnormal signs and laboratory examination index returned to normal; Partial response (PR)-most of clinical symptoms, more than two kinds of abnormal laboratory indexes improve; Stable disease (Stable disease)-clinical symptoms and abnormal signs and laboratory examination index does not increase, and the improvement is not obvious; Disease progression (PD)clinical symptoms and abnormal signs and laboratory examination indexes increase gradually. The calculation formula of total efficiency is: total efficiency $=(\mathrm{CR}+\mathrm{PR}) /$ total number of people $\times 100 \%$. Before statistical analysis of quantitative data, Kolmogorov-Smirnov was used for normality test. Sig. $>0.05$ followed normal distribution, otherwise it was non-normal distribution. Quantitative data conforming to normal distribution were expressed as mean \pm standard deviation $(\overline{\mathrm{x}} \pm \mathrm{s})$ and differences were analyzed by $t$ test. The non-normal distribution quantitative data were represented by the median $\left(\mathrm{P}_{25}, \mathrm{P}_{75}\right)$ and the difference was analyzed by rank sum test. Qualitative data was expressed as percentage (\%) and difference analysis was conducted by chi-square test. $\mathrm{p}<0.05$ was considered statistically significant. All the above data were analyzed by Statistical package for the social sciences (SPSS) 22 software. The general clinical data are shown in the table below: in the control group, there were 27 patients (age $58.4 \pm 12.2$ y old), including 14 males and 13 females, and 9 patients with hyperc immunoglobulinemia. According to Ann Arbor staging, there were 1 patient with stage I, 10 patients with stage II, 7 patients with stage III, and 9 patients with stage IV. In the treatment group, there were 62 patients (age $56.3 \pm 14.2$ y old), including 25 males and 37 females, and 18 patients with hypergamma immunoglobulinemia. According to the Ann Arbor staging, 7 cases were stage I, 16 cases were stage II, 10 cases were stage III and 29 cases were stage IV. There were no significant differences in age, gender, incidence of hypergamma $\mathrm{C}$ immunoglobulinemia, International Prognostic Index (IPI) score, tumor stage and previous medical history between these two groups, as shown in Table 1. The changes of biochemical indexes before and after treatment were analyzed based on Mann-Whitney $U$ test, there was no statistical difference in the levels of hemoglobin, erythrocytic sedimentation rate, albumin,
LDH and $\beta 2$ microglobulin between the control group and the treatment group before and after treatment $(p>0.05)$. The rate of erythrocyte sedimentation rate in the treatment group and the control group after treatment with rituximab combined with chemotherapy was lower than that before treatment, with statistical difference $(p<0.05)$, as shown in Table 2 . The changes of immunoglobulin levels before and after treatment were analyzed, there was no statistical difference in $\mathrm{IgG}$, IgA, IgM and globulin levels between the control group and the treatment group before treatment $(\mathrm{p}>0.05)$. After treatment, the level of globulin in the treatment group was significantly lower than that in the control group, with statistical difference $(p<0.05)$. Within groups, the levels of $\operatorname{IgG}, \operatorname{IgA}, \operatorname{IgM}$ and globulin in the treatment group after treatment with rituximab combined with chemotherapy were lower than before treatment, with statistical differences $(p<0.05)$. The levels of $\mathrm{IgG}, \mathrm{IgA}$ and globulin in the control group were significantly lower after treatment than before $(p<0.05)$ and there was no significant difference in IgM levels before and after treatment $(\mathrm{p}>0.05)$; Table 3 . After traditional chemotherapy plus rituximab, the total effective rate of the treatment group was $82.3 \%$, which was much higher than that of the control group using traditional chemotherapy $(55.6 \%)$ and the difference was statistically significant $(\mathrm{p}<0.05)$, as shown in Table 4. In the treatment group, the total effective rate of patients with hypergamma globulinemia after rituximab combing with chemotherapy was $85.4 \%$, which was much higher than the normal group with normal gamma globulin level $(26.8 \%)$ and the difference was statistically significant $(\mathrm{p}<0.05)$, as shown in Table 5 . DLBCL is a common malignant lymphoma. Its etiology is closely related to viral infection, bacterial infection, immune system deficiency, bad living habit and its pathological mechanism is complex. Traditional chemotherapy has not a satisfying therapeutic effect on most patients. A large number of domestic and foreign studies have reported that clonal antibody (rituximab) combined with anthracycline chemotherapy has achieved a good therapeutic effect with low adverse reactions, greatly improving the prognosis of patients $^{[11-13]}$. DLBCL expressed a variety of B cell antigens, such as cases positive (CD) CD19, CD22, CD45, CD20, etc., and CD20 was expressed in more than $90 \%$ of them. Rituximab could specifically bind to CD20 to reduce cancer cell proliferation and accelerate apoptosis. Compared with conventional chemotherapy, this study found that the total effective rate was $74.2 \%$ and the complete remission rate was $58.1 \%$ in patients 
TABLE 1: COMPARISON OF GENERAL CLINICAL DATA $(\mathbf{n}=\mathbf{8 9})$

\begin{tabular}{|c|c|c|c|c|}
\hline Index & Control group & Treatment group & $t / \chi^{2}$ & $\mathbf{P}$ \\
\hline Case $(n, \%)$ & $27(30.3 \%)$ & $62(69.7 \%)$ & - & - \\
\hline Age $\left({ }^{-} \pm s\right)$ & $58.4 \pm 12.2$ & $56.3 \pm 14.2$ & 0.646 & 0.520 \\
\hline \multicolumn{5}{|l|}{ Gender (n, \%) } \\
\hline Male & $14(51.9 \%)$ & $25(40.3 \%)$ & \multirow{2}{*}{1.016} & \multirow{2}{*}{0.314} \\
\hline Female & $13(48.1 \%)$ & 37 (59.7 \%) & & \\
\hline \multicolumn{5}{|c|}{ Gamma immunoglobulinemia (n, \%) } \\
\hline High & $13(14.6 \%)$ & $41(46.1 \%)$ & \multirow{3}{*}{8.138} & \multirow{3}{*}{0.017} \\
\hline Normal & $11(12.4 \%)$ & $21(23.6 \%)$ & & \\
\hline Low & $3(3.4 \%)$ & $0(0.0 \%)$ & & \\
\hline \multicolumn{5}{|l|}{ IPI score } \\
\hline low risk 0 1 & $6(22.2 \%)$ & $15(24.2 \%)$ & \multirow{3}{*}{3.755} & \multirow{3}{*}{0.440} \\
\hline middle risk 2 3 & $17(63.0 \%)$ & $36(58.1 \%)$ & & \\
\hline high risk 4 5 & $4(14.8 \%)$ & $11(17.7 \%)$ & & \\
\hline \multicolumn{5}{|l|}{ Tumor stage $(\mathrm{n}, \%)$} \\
\hline I a & $(0.0 \%)$ & $7(11.3 \%)$ & \multirow{9}{*}{17.438} & \multirow{9}{*}{0.258} \\
\hline I b & $1(3.7 \%)$ & $(0.0 \%)$ & & \\
\hline II a & 7 (25.9\%) & $13(21.0 \%)$ & & \\
\hline II $\mathrm{b}$ & $3(11.1 \%)$ & $3(4.8 \%)$ & & \\
\hline III a & 1 (3.7 \%) & $8(12.9 \%)$ & & \\
\hline III b & $5(18.5 \%)$ & $2(3.2 \%)$ & & \\
\hline III c & $1(3.7 \%)$ & $(0.0 \%)$ & & \\
\hline IV a & $6(22.2 \%)$ & $15(24.2 \%)$ & & \\
\hline $\mathrm{IV} \mathrm{b}$ & $3(11.1 \%)$ & $14(22.6 \%)$ & & \\
\hline \multicolumn{5}{|l|}{ Previous medical history (n, \%) } \\
\hline Hepatitis C & $(0.0 \%)$ & $3(4.8 \%)$ & \multirow{16}{*}{31.140} & \multirow{16}{*}{0.119} \\
\hline Hypertension & $4(14.8 \%)$ & $8(12.9 \%)$ & & \\
\hline Diabetes & $2(7.4 \%)$ & $3(4.8 \%)$ & & \\
\hline Coronary heart disease (CHD) & $2(7.4 \%)$ & $2(3.2 \%)$ & & \\
\hline Thyroid neoplasm & $1(3.7 \%)$ & $1(1.6 \%)$ & & \\
\hline Cerebral infarction & $(0.0 \%)$ & $1(1.6 \%)$ & & \\
\hline Myocardial infarction (ami) & $1(3.7 \%)$ & $1(1.6 \%)$ & & \\
\hline Hepatitis B & $2(7.4 \%)$ & $4(6.5 \%)$ & & \\
\hline Sjogren's syndrome & $(0.0 \%)$ & $1(1.6 \%)$ & & \\
\hline Chronic kidney disease & $(0.0 \%)$ & $1(1.6 \%)$ & & \\
\hline Epilepsy & $1(3.7 \%)$ & $(0.0 \%)$ & & \\
\hline Tuberculosis (TB) & $1(3.7 \%)$ & $(0.0 \%)$ & & \\
\hline Crohn's disease & $1(3.7 \%)$ & $(0.0 \%)$ & & \\
\hline Rheumatoid & $1(3.7 \%)$ & $(0.0 \%)$ & & \\
\hline Evans syndrome & $1(3.7 \%)$ & $(0.0 \%)$ & & \\
\hline Pneumonia & 1 (3.7 \%) & $1(1.6 \%)$ & & \\
\hline
\end{tabular}

TABLE 2: CHANGES OF IMMUNOGLOBULIN LEVELS BEFORE AND AFTER TREATMENT

\begin{tabular}{lllllll}
\hline Group & & Hemoglobin $(\mathrm{g} / \mathrm{L})$ & ESR $(\mathrm{mm} / \mathrm{h})$ & Albumin $(\mathrm{g} / \mathrm{L})$ & LDH $(\mathrm{U} / \mathrm{L})$ & $\mathrm{B}_{2}$ microglobulin $(\mathrm{mg} / \mathrm{L})$ \\
\hline Control & Before treatment & $124.5(104.3,150)$ & $43(16,121)$ & $38(32.8,44.1)$ & $207(132,461)$ & $2.99(1.78,4.18)$ \\
group & After treatment & $121.9(87.1,155)$ & $21(11,78)^{*}$ & $42.3(32.8,48.2)$ & $208(128,562)$ & $2.63(1.64,4.46)$ \\
Treatment & Before treatment & $121.3(99,140)$ & $44.5(22,83)$ & $41.25(36.1,44.4)$ & $220(160,392)$ & $2.51(1.85,4.3)$ \\
group & After treatment & $116(101,143)$ & $20(13,36)^{*}$ & $42.9(35.7,47.1)$ & $211(164,326)$ & $2.435(1.7,4.03)$ \\
\hline
\end{tabular}

Note: Compared with before treatment, ${ }^{*} \mathrm{p}<0.05$; compared with control group, ${ }^{\#} p<0.05$.

treated with rituximab. Rituximab can bind with immune cells or complement component 1q (C1q), which is conducive to the recognition and killing of cancer cells by immune cells. However, C1q can bind to the $\mathrm{CH} 2$ site of IgG molecule to form immune complex, and when $\mathrm{Clq}$ binds to IgG or IgM, classical 
TABLE 3: CHANGES OF IMMUNOGLOBULIN LEVELS BEFORE AND AFTER TREATMENT

\begin{tabular}{lccccc}
\hline Group & & Hemoglobin $(\mathrm{g} / \mathrm{L})$ & $\operatorname{lgA}(\mathrm{g} / \mathrm{L})$ & $\operatorname{lgM}(\mathrm{g} / \mathrm{L})$ & Globulin $(\mathrm{g} / \mathrm{L})$ \\
\hline \multirow{2}{*}{ Control group } & Before treatment & $15.20(12.10,28.70)$ & $3.61(1.72,6.36)$ & $1.52(0.5,4.38)$ & $32.3(29.2,53.3)$ \\
& After treatment & $12.50(9.70,14.80)^{*}$ & $2.78(1.89,3.89)^{*}$ & $1.56(0.76,5.63)$ & $29.7(24.3,37.8)^{*}$ \\
\multirow{2}{*}{ Treatment } & Before treatment & $18.35(13.20,22.40)$ & $4.02(2.06,5.75)$ & $1.535(0.75,4.87)$ & $33.4(29.3,41.7)$ \\
group & After treatment & $11.10(8.00,14.30)^{*}$ & $2.41(1.59,3.22)^{*}$ & $1.07(0.55,2.09)^{*}$ & $26.2(22.4,30)^{*}$ \\
\hline
\end{tabular}

Note: Compared with before treatment, ${ }^{*} \mathrm{p}<0.05$; compared with control group, ${ }^{\#} \mathrm{p}<0.05$.

TABLE 4: CLINICAL EFFICACY ANALYSIS

\begin{tabular}{|c|c|c|c|c|c|}
\hline \multirow{2}{*}{ Group } & \multicolumn{4}{|c|}{ Efficacy } & \multirow{2}{*}{ Total effective rate } \\
\hline & CR & PR & Stable disease & Disease progression & \\
\hline Control group & $10(37.0 \%)$ & 4 (14.8\%) & $311.2 \%)$ & $10(37.0 \%)$ & $51.9 \%$ \\
\hline Treatment group & $36(58.1 \%)$ & $10(16.1 \%)$ & $14(22.6 \%)$ & $2(3.2 \%)$ & $74.2 \% \#$ \\
\hline$\chi^{2}$ & & & & & 4.274 \\
\hline $\mathrm{p}$ & & & & & 0.039 \\
\hline
\end{tabular}

Note: Compared with control group, ${ }^{*} \mathrm{p}<0.05$.

TABLE 5: INFLUENCE OF HYPERGAMMA GLOBULINEMIA ON RITUXIMAB

\begin{tabular}{lccccc}
\hline \multirow{2}{*}{ Group } & \multicolumn{3}{c}{ Efficacy } & Total effective rate \\
\cline { 2 - 5 } Hypergamma globulinemia & CR & PR & Stable disease & Disease progression \\
Normal group & $6(73.2 \%)$ & $5(12.2 \%)$ & $5(12.2 \%)$ & $1(2.4 \%)$ & $85.4 \% \#$ \\
$\chi 2$ & & $5(12.2 \%)$ & $9(22.0 \%)$ & $1(2.4 \%)$ & $26.8 \%$ \\
$\mathrm{p}$ & & & & 7.891 & 0.00497 \\
\hline
\end{tabular}

Note: Compared with normal group, ${ }^{\#}$ means $\mathrm{p}<0.05$.

complement activation pathway will be initiated ${ }^{[14]}$. Patients with hypergamma globulinemia have higher IgG levels and complement dependent cytoxicity (CDC) of rituximab is enhanced by complement activation $^{[15]}$. In this study, patients with hypergamma globulinemia treated with rituximab had a total effective rate of $85.4 \%$, better than patients with normal gamma globulinemia. Therefore, rituximab combing with chemotherapy has an obvious effect on diffuse large B-cell lymphoma complicated with hypergamma globulinemia, which can effectively reduce the level of immunoglobulin in vivo, providing a reference for clinical treatment.

\section{Acknowledgements:}

This work was supported by Clinical features and prognostic analysis of lymphoproliferative diseases with hypergammaglobulinemia (No. 20160655).

\section{Conflicts of interest:}

The authors report no conflicts of interest.

\section{REFERENCES}

1. Dispenzieri A, Gertz MA, Therneau TM, Kyle RA. Retrospective cohort study of 148 patients with polyclonal gammopathy. Mayo Clin Proc 2001;76:476-87.

2. Zou L, Chen G, Zhou Y, Ye W, Wen Y, Chen L, et al. Continuous hypergammaglobulinemia and proteinuria after the recovery of the visceral Leishmaniasis: a case report. BMC Infect Dis 2021;21(1):1-5.

3. Huo L, Song L, Li N, Li X. A Case of Immunoglobulin G4-Related Autoimmune Pancreatitis with Extreme Hypergammaglobulinemia. Pancreas 2020;49(5):e39.

4. Yang M, Xie JP. A case of Sjogren's syndrome with multiple bullae and polyclonal hypergammaglobulinemia. J Clin Intern Med 2020;37(01):65-66.

5. Siegel RL, Miller KD, Jemal A. Cancer statistics, 2019. CA Cancer J Clin 2019;69(1):7-34.

6. Huang L, Chen LJ, Jiang XM. New progress in the research of malignant lymphoma in 2019. Evid Based Med 2020;20(1):218.

7. Huang X. Clinical observation of rituximab combined with chemotherapy in the treatment of diffuse large B-cell lymphoma. Electronic J Clin Med Literature 2019;6(28):1445.

8. He JH, Zhao R. The effect of rituximab injection combined with chemotherapy on the clinical efficacy of diffuse large B-cell lymphoma and the effect of serum CRP, TNF- $\alpha$ and CA125 levels. Youjiang Med 2021;49(01):42-5.

9. Li JM, Guan ZZ, Shen T. Guidelines for the diagnosis and treatment of diffuse large B-cell lymphoma in China (2013 edition). Chin J Hematol 2013;34(09):816-9.

10. Li WY, Huang FJ, Lu XH. To explore the efficacy of rituximab 
monoclonal antibody combined with CHOP regimen in the treatment of patients with diffuse large B-cell lymphoma. Clin Res 2019;27(09):98-9.

11. Adams HJ, Nievelstein RA, Kwee TC. Prognostic value of complete remission status at end-of-treatment FDG-PET in R-CHOP-treated diffuses large B-cell lymphoma: systematic review and meta-analysis. Br J Haematol 2015;170(2):185-91.

12. Utsu Y, Takaishi $K$, Inagaki $S$, Arai H, Yuasa H, Masuda $S$, et al. Influence of dose reduction of vincristine in R-CHOP on outcomes of diffuse large B cell lymphoma. Ann Hematol 2016;95(1):41-7.

13. Yu S, Luo H, Pan M, Palomino LA, Song X, Wu P, et al. High frequency and prognostic value of MYD88 L265P mutation in diffuse large B-cell lymphoma with R-CHOP treatment. Oncol Lett 2018;15(2):1707-15.

14. Yumi K. Immune Complex Assay Test (C1q-IgG, C3dIgG) Discussion. Ehime Prefectural Clin Lab Technician
Association J 1991;37.

15. Winkler MT, Bushey RT, Gottlin EB, Campa MJ, Guadalupe ES, Volkheimer AD, et al. Enhanced CDC of B cell chronic lymphocytic leukemia cells mediated by rituximab combined with a novel anti-complement factor $\mathrm{H}$ antibody. Plos one 2017;12(6):e0179841.

This is an open access article distributed under the terms of the Creative Commons Attribution-NonCommercial-ShareAlike 3.0 License, which allows others to remix, tweak, and build upon the work non-commercially, as long as the author is credited and the new creations are licensed under the identical terms

This article was originally published in a special issue,

"Evolutionary Strategies in Biomedical Research and

Pharmaceutical Sciences" Indian J Pharm Sci 2021:83(3)

Spl issue;114-119 